\title{
Electronic "photoreceptors" enable prosthetic vision with acuity matching the natural resolution in rats
}

\section{Bing-Yi Wang ${ }^{1},{ }^{*}$, Zhijie Charles Chen ${ }^{2},{ }^{*}$, Mohajeet Bhuckory ${ }^{3,4}$, Tiffany Huang ${ }^{2}$, Andrew Shin ${ }^{5}$, Valentina Zuckerman ${ }^{3}$, Elton Ho Ho $^{3,4}$, Ethan Rosenfeld ${ }^{3}$, Ludwig Galambos ${ }^{2}$, Theodore Kamins ${ }^{2,3}$, Keith Mathieson ${ }^{6}$, and Daniel Palanker ${ }^{3,4}$}

${ }^{1}$ Department of Physics, Stanford University, Stanford, CA, USA

${ }^{2}$ Department of Electrical Engineering, Stanford University, Stanford, CA, USA

${ }^{3}$ Hansen Experimental Physics Laboratory, Stanford University, Stanford, CA, USA

${ }^{4}$ Department of Ophthalmology, Stanford University, Stanford, CA, USA

${ }^{5}$ Department of Material Science, Stanford University, Stanford, CA, USA

${ }^{6}$ Department of Physics, Institute of Photonics, University of Strathclyde, Glasgow, Scotland, UK

* These authors contributed equally to this work.

\begin{abstract}
Localized stimulation of the inner retinal neurons for high-acuity prosthetic vision requires small pixels and minimal cross-talk from neighboring electrodes. Local return electrodes within each pixel limit crosstalk, but can over-constrain the electric field, thus precluding efficient stimulation with subretinal pixels smaller than 50 $\mu \mathrm{m}$. Here we demonstrate high-resolution prosthetic vision based on a novel design of a photovoltaic array, where field confinement is achieved dynamically, leveraging the adjustable conductivity of the diodes under forward bias to turn the designated pixels into transient returns. We validated computational modeling of the field confinement in such an optically-controlled circuit by ex-vivo and in-vivo measurements. Most importantly, using this strategy, we demonstrated that the grating acuity with $40 \mu \mathrm{m}$ pixels matches the pixel pitch, while with $20 \mu \mathrm{m}$ pixels, it reaches the $28 \mu \mathrm{m}$ limit of the natural visual resolution in rats. This method enables customized field shaping based on individual retinal thickness and distance from the implant, paving the way to prosthetic vision with acuity as high as 20/80 in atrophic macular degeneration.
\end{abstract}

\section{Introduction}

Retinal degenerative diseases, such as age-related macular degeneration (AMD) and retinitis pigmentosa, are a leading cause of untreatable visual impairment and legal blindness ${ }^{1,2}$. Despite the irreversible loss of photoreceptors, the inner retinal neurons survive to a large extent ${ }^{3,4}$. Electrical stimulation of the secondary retinal neurons, mainly the bipolar cells, elicits visual percepts ${ }^{5,6}$, hence enabling electronic restoration of sight (Figure 1). AMD patients with a photovoltaic subretinal implant PRIMA (Pixium Vision, Paris, France) having bipolar pixels of $100 \mu \mathrm{m}$ in width (corresponding to Snellen acuity of 20/420) demonstrated a prosthetic letter acuity of $1.17 \pm 0.13$ pixels, corresponding to the Snellen range of 20/460-20/565 $5^{5,7}$. Even though this is an exciting proof of concept, for a wide adoption of this approach by AMD patients, prosthetic acuity should significantly exceed their remaining peripheral vision, which is typically no worse than 20/400. The sampling limit for an acuity of 20/200 (US legal blindness threshold) corresponds to $50 \mu \mathrm{m}$ pixels, while 20/100 requires $25 \mu \mathrm{m}$ pixels.

As with natural vision, prosthetic visual acuity is fundamentally limited not only by the spatial resolution of the stimulation patterns (i.e. pixel size and the electric field spread in retinal tissue), but also by their contrast, which is affected by crosstalk between the neighboring electrodes ${ }^{8}$. The lateral spread of an electric field can be confined by local return electrodes in each pixel, as in the PRIMA implant. However, scaling down such pixels is difficult because the penetration depth of the electric field in tissue is constrained by the distance between the active and return electrodes, which is about half of the pixel radius (Figure $2 \mathrm{~A}$ ). As a result, the retinal stimulation threshold in such a geometry rapidly increases with decreasing pixel size, and exceeds the charge injection limit of even one of the best electrode materials (sputtered iridium oxide film; SIROF) when pixel sizes fall below $40 \mu \mathrm{m}^{9}$.

One approach to overcoming this problem is based on elevating the return electrode to the top of the inner nuclear layer using a 3-D honeycomb-shaped array, thereby orienting the electric field vertically within the 
wells (Figure $2 \mathrm{~B})^{9}$. This arrangement decouples the field penetration depth from the pixel width and greatly reduces the stimulation threshold because the vertical field matches the orientation of the bipolar cells in the retina ${ }^{9}$. Although initial animal studies have shown promising results with retinal migration into the subretinal wells, functional activity of the migrated neurons into 3-D arrays has still to be confirmed. In addition, the fabrication process of the honeycomb structures with a local return electrode needs further development.

Here, we present an alternative approach to high-resolution prosthetic vision with a planar monopolar subretinal implant ${ }^{10}$, where the high penetration depth and high contrast are enabled by the spatiotemporal modulation of the pixels, utilizing the exponentially changing conductivity of the photodiodes under bias. In our approach, images captured by a camera are projected onto the subretinal photovoltaic arrays from the augmented-reality glasses using pulsed near-infrared $(880 \mathrm{~nm})$ light ${ }^{5,7}$. Photodiodes in each pixel convert the incident photons into pulsed electric current flowing through the tissue between the active and return electrodes on the array. Stimulated bipolar cells transmit the visual information via the retinal neural network to the brain. Accumulation of charge at the electrode-electrolyte interface and coupling of the electric potentials from the neighboring pixels in the electrolyte can elevate the voltage on the active electrodes. Since the active electrode is connected to the $p+$ contact of the diode and the return electrodes connect to the $n+$ contact in our pixels, the diode becomes forward biased when positive charge accumulates on interface of the active electrode with electrolyte. Therefore, pre-charging some of the active electrodes using proper illumination protocol can make them sufficiently conductive to serve as effective return electrodes for the next image frame, when these pixels are no longer illuminated and others are activated. The distance between the active electrode and the neighboring return electrode affects the penetration depth of the electric field into the tissue (Figure 2C). Such pre-conditioning of the pixels to become transient returns in the next image frame enables flexible control of the lateral and axial confinement of electric field in tissue by spatiotemporal modulation of the images projected onto the photovoltaic array. This optical current steering strategy potentially allows optimization of the stimulation depth and lateral selectivity for every patient, depending on the retinal thickness and its proximity to the implant ${ }^{11}$.

To model this mode of operation, we first characterized the spatial coupling of electric potential among the pixels using a static finite-element method, and then computed the circuit dynamics of the photovoltaic array, taking into account the inter-pixel coupling, from which the dynamics of the potential distribution in the electrolyte is derived. The computational results are then validated by comparison to ex-vivo measurements of the electric potential in the electrolyte, as well as to electrograms recorded on the corneas of rats implanted with photovoltaic arrays. Most importantly, we demonstrate for the first time that prosthetic acuity in blind rats with subretinal implants composed of $40 \mu \mathrm{m}$ pixels matches the pixel pitch, while with $20 \mu \mathrm{m}$ pixels, it reaches the 28 $\mu \mathrm{m}$ limit of their natural visual resolution.

\section{Results}

\section{Computational modeling of electric field in tissue}

The charge accumulated at the electrode-electrolyte interface of a photovoltaic pixel discharges by forward-biased conduction through the diode, driven collectively by the potential at the electrode-electrolyte capacitance due to charge accumulation and the effect of electric potentials from the neighboring pixels. When the forward current through the diode becomes comparable to the stimulation current of the neighboring pixels, such a discharging pixel serves as a transient return. Such a local return electrode limits the spread of electric fields from adjacent pixels, reducing the crosstalk and limiting the field penetration into the tissue (Figure $2 \mathrm{C}$ ). The cross-pixel resistance between two adjacent electrodes can be defined as $R_{1,2}=V_{1,2} / I_{1}$, where $I_{1}$ is the current injection of electrode 1 , and $V_{1,2}$ is the potential rise at electrode 2 caused by $I_{1}$. The finite-element model (COMSOL) of the subretinal photovoltaic array with $40 \mu \mathrm{m}$ pixels yields $R_{1,2}$ in the range of 15 to $30 \mathrm{k} \Omega$, depending on the location of the electrodes in the array. Each pixel in the hexagonal mesh is surrounded by 6 neighbors, and the stimulation current is typically between 0.1 and $1 \mu \mathrm{A}$. Therefore, summation of these electric potentials in the electrolyte during simultaneous activation of multiple pixels, together with accumulation of charge at the capacitive electrode-electrolyte interface during previous pulses, may elevate the electrode potential close to the turn-on voltage of the Si diode (about 0.5 V). 
Figure 3 shows the effect of such transient returns on a grating pattern, with every other line of the $40 \mu \mathrm{m}$ pixels activated by illumination at $1 \mathrm{~mW} / \mathrm{mm}^{2}$. In this calculation, we assumed exact alignment of the light pattern to the pixels. Misalignment will cause some smearing and decrease in contrast, varying over time due to the eye movements. Since the activation function of the bipolar cell stimulation can be closely approximated by a trans-cellular voltage step (between the dendritic and axonal ends of a neuron) ${ }^{12}$, we plot a potential difference between $10 \mu \mathrm{m}$ above the implant (bottom of the BC dendrites) to $57 \mu \mathrm{m}$ - the middle of the IPL, where the axonal terminals of the average $\mathrm{BC}$ are located ${ }^{9}$, as illustrated in Figure $2 \mathrm{~A}$. When the electrode-electrolyte interfaces of the dark pixels are pre-charged to $0.5 \mathrm{~V}$ prior to the light pulse, they become sufficiently conductive to drain the current during the next pulse and hence the potentials in the electrolyte above the dark pixels drop below $0 \mathrm{~V}$, thereby providing $100 \%$ contrast (Figure 3B,D). On the contrary, if the electrochemical potential bias is far below the turn-on voltage of the diode (e.g. $0.2 \mathrm{~V}$ ) the dark pixels are not conductive and hence do not transform into transient returns. Therefore, the contrast is reduced to below $30 \%$ as a result of a strong crosstalk from the neighboring pixels (Figure 3B,C). Contrast decreases even further if the array is composed of smaller pixels, and also declines with distance from the implant. Such low contrast precludes high-resolution prosthetic vision in general, and grating acuity measurements, in particular.

\section{Experimental validation of the model}

We recorded the electric potential, generated by the photovoltaic array in Dulbecco's phosphate-buffered saline (DPBS) under $10 \mathrm{~ms} \mathrm{NIR}(880 \mathrm{~nm})$ pulses repeated at $40 \mathrm{~Hz}$, using a pipette electrode positioned $20 \mu \mathrm{m}$ above a pixel in a Petri dish. First, the device was driven to the steady-state under uniform full-field illumination at a peak irradiance of $8 \mathrm{~mW} / \mathrm{mm}^{2}$ (Figure 4A). Then a field stop in the projection system was rapidly closed between the two laser pulses, restricting the illumination to an octagonal area of about $1 \mathrm{~mm}$ in width, as shown in Figure 4B,C. The electric potential outside the field stop was recorded during the transition into the post-closure steady-state. Although the recorded pixel in the shadowed area does not inject current after the closure, positive pulses are still detectable in the post-closure steady-state due to crosstalk from the illuminated pixels. However, during the transition period between the post-closure state and the steady-state, instead of a positive pulse, a negative dip was observed at the onset of the first light pulse after the closure (Figure $4 \mathrm{D}, \mathrm{t}=0 \mathrm{~ms}$ ), showing that the pixel begins to sink current and demonstrating the crosstalk suppression by the transient returns - as predicted by our model.

To further validate the spatiotemporal model in an implanted rat eye, we calculated the electrical signal generated by the implant at the cornea for various pulse repetition rates, ranging from 1 to $125 \mathrm{~Hz}$ with full-field illumination and with alternating gratings. For this purpose, we created a computational model of the rat's eye ${ }^{13}$ and a head, with the photovoltaic array implanted in the subretinal space (Figure 5A), as described in detail in Methods and the Supplementary Materials. The modeling results were compared with the in-vivo measurements of the corneal potential relative to a reference electrode in the nose. With a full-field illumination at $1.2 \mathrm{~mW} / \mathrm{mm}^{2}$ of $4 \mathrm{~ms}$ in duration repeated at $1 \mathrm{~Hz}$, the amplitude of the corneal potential was in the range of 2 to $3.5 \mathrm{mV}$, varying between animals and with the position of the electrode on the cornea. As shown in Figure 5B, modeling closely matches the shape of the measured corneal signal. Due to the variation of the corneal signals across animals, we normalized the frequency dependence plot by the amplitude at $1 \mathrm{~Hz}$ before averaging. As shown in Figure $5 \mathrm{C}$, the frequency dependence of the corneal signal also closely matches the model. Amplitude of the visually evoked potentials (VEPs) decreases with increasing frequency much faster than the corneal signals, dropping to the noise level near $60 \mathrm{~Hz}$ (flicker fusion). For this reason, we performed the acuity measurements at a pulse repetition rate of $64 \mathrm{~Hz}$, as described in the next section.

\section{Grating acuity in natural and prosthetic vision}

Measurements of the VEPs in response to alternating gratings is a common method of assessing vision in animal models ${ }^{14}$ or infants ${ }^{15}$. We recorded VEPs via transcranial electrodes above the visual cortices in Royal College of Surgeons (RCS) rats having subretinal photovoltaic implants and in Long Evans (LE) rats, as a normally-sighted control. The corneal signal generated by the implant was also recorded for characterization of the implant's current injection. For prosthetic vision, grating images were delivered with NIR light $(880 \mathrm{~nm})$ at 1.2 $\mathrm{mW} / \mathrm{mm}^{2}$ peak irradiance at the retina using $4 \mathrm{~ms}$ pulses at $64 \mathrm{~Hz}$ repetition rate, while the pattern reversal occurred every $500 \mathrm{~ms}$ (Figure 6). We evaluated the prosthetic visual acuity with implants having $40 \mu \mathrm{m}$ and $20 \mu \mathrm{m}$ 
pixels. For the natural visual acuity measurements, images of the black-and-white gratings presented on a screen under continuous illumination also alternated every $500 \mathrm{~ms}$.

The prosthetic VEP waveforms contained both a $2-\mathrm{Hz}$ signature induced by the grating reversal, and a $64-\mathrm{Hz}$ component of the carrier frequency, including the stimulus artifact. We used the corneal signal as a template to remove the artifact and filtered out the high frequency components. Amplitude of the grating reversal response was measured as the peak-to-peak voltages in the VEP waveform between 0 and $100 \mathrm{~ms}$. The natural grating acuity is typically defined in angular resolution units, i.e. cycles per degree (cpd), and is determined in electrophysiological measurements by the intersection of the logarithmic fitting line with the noise level in a log-linear plot $^{16,17}$. We followed this convention (Figure 7), but to relate the resolution to the pixel size, we plotted the VEP amplitude vs. the inverse bar width (half a cycle). In this conversion, we utilized the fact that one degree of the visual angle corresponds to $64.3 \pm 2.9 \mu \mathrm{m}$ on the retina, as described in Methods. Corresponding plots in the linear units of bar widths are shown in Supplementary Figure S5.

For prosthetic vision with $40 \mu \mathrm{m}$ implants, the visual acuity was found to be $34.3 \pm 5.5 \mu \mathrm{m}$, matching the pixel pitch in a hexagonal array $p=40 \mu \mathrm{m} \cdot \cos \left(30^{\circ}\right)=34.6 \mu \mathrm{m}$. However, with $20 \mu \mathrm{m}$ pixels the grating acuity did not match the pixel pitch of $17.3 \mu \mathrm{m}$. Instead, it was $27.1 \pm 3.8 \mu \mathrm{m}$, matching the natural grating acuity of $27.9 \pm$ $2.8 \mu \mathrm{m}$, measured in LE rats, as shown in Figure 7 .

\section{Discussion}

All previous studies with subretinal photovoltaic implants showed that the prosthetic visual acuity matches the pixel pitch $\left(100 \mu \mathrm{m}\right.$ in humans ${ }^{5}, 75$ and $55 \mu \mathrm{m}$ in rats $\left.{ }^{18,19}\right)$, just as the case with our $40 \mu \mathrm{m}$ pixels in this study. Here, however, we demonstrated that prosthetic visual acuity with $20 \mu \mathrm{m}$ pixels is not limited by the pixel pitch, but rather matches the $28 \mu \mathrm{m}$ natural visual acuity limit in rats, indicating that with such small pixels, resolution appears to be limited by the retinal network integration in rats rather than by the sensor size. However, in humans, where the $20 / 20$ vision corresponds to $5 \mu \mathrm{m}$ stripes on the retina, we hope that $20 \mu \mathrm{m}$ pixels will enable restoration of central vision in AMD patients with a visual acuity as high as 20/80. Restoration of visual acuity at this level would be of major clinical significance for patients blinded by advanced AMD.

An electric field around the pixels could be optimally confined by adjusting the distance between the active and return electrodes. Such optical configurability of the electric field enables optimization of the field penetration depth for individual patients, tailored to their particular retinal thickness and the implant's proximity to bipolar cells ${ }^{20}$. At the same time, properly restricting the field penetration depth may avoid the direct activation of the tertiary retinal neurons: the amacrine and ganglion cells. Ideally, this could be accomplished if each pixel would have an independent control of its conductivity at any moment in time. This could be done, for example, by including a photosensitive transistor connected in parallel with a primary photodiode in each pixel, which would respond to a wavelength different from the primary NIR light. However, this would greatly complicate the implant design and fabrication. Here we describe a much more practical approach to such field confinement.

The monopolar photovoltaic array, having a common return electrode at the edge of the implant or on its back, allows the use of some of the active electrodes as anodes and some as cathodes, depending on their pre-charging history and the light intensity on each pixel. Due to charge accumulation during previous exposures, the electrode potential stays close to the diode turn-on voltage, so its forward current balances the photocurrent corresponding to the average light intensity. Additional pre-conditioning of the photovoltaic pixels in real time can be based on images captured by the camera. For a typical image refresh rate of $30 \mathrm{~Hz}$, each frame lasts about 33 ms. Photovoltaic stimulation pulses typically range from 0.8 to $10 \mathrm{~ms}^{5}$, leaving at least $23 \mathrm{~ms}$ for the pre-charging phase preceding the stimulation phase in each frame. Upon image acquisition, the sufficiently dark pixels will be designated to become the local returns and will be exposed to light below the stimulation threshold to accumulate additional charge prior to activation of the bright pixels in the image. Then, during the stimulation phase, the brighter pixels are illuminated with intensity and duration corresponding to the desired charge injection, while the pre-charged dark pixels sink the current injected from the nearby active pixels. This pre-conditioning strategy has the potential to achieve field confinement and hence a high spatial resolution, with a delay not exceeding one frame relative to the image acquisition. To accelerate the image processing for determining which pixels need to be pre-conditioned, predictive tracking algorithms, such as the Kalman filter or exponential smoothing, could be applied. This approach may also allow the stretching the pre-charging phase over several frames. 
Another strategy for reducing the cross-talk could be a sequential stimulation spread over the whole 33 $\mathrm{ms}$ of the frame duration. With the average pulse duration of about $4 \mathrm{~ms}$, only about $4 / 33=12 \%$ of the pixels can be activated simultaneously, and even fewer than that, if the images are sparse. In such a distributed activation, pixels become the transient returns after their light is turned off, while other pixels are turned on. However, with a stimulation spread over the frame duration eye movements may shift the projected image and introduce some uncertainty regarding the relative location of the active and return electrodes. During the slow drift $(<0.25 \mathrm{deg} / \mathrm{s})$, the $2.5 \mu \mathrm{m} /$ frame image shift is negligible compared to the $20 \mu \mathrm{m}$ pixel size, but it becomes noticeable during microsaccades, which occur $1-2$ times a second ${ }^{21}$. On average, the eye movement velocity is about $1 \mathrm{deg} / \mathrm{s}^{22}$, corresponding to about $10 \mu \mathrm{m} /$ frame at $30 \mathrm{~Hz}$ frame rate, which imposes a lower limit on pixel size for predictable activation, unless eye tracking is employed or frame rate is increased to reduce this uncertainty.

In conclusion, we have demonstrated prosthetic vision with a resolution matching the natural acuity in rats, using a novel design of a photovoltaic array, where electric field confinement is achieved dynamically, by turning the designated pixels into transient returns. This method enables customized field shaping for each patient and paves the way to prosthetic vision with an acuity exceeding 20/100.

\section{Methods \\ Modeling and ex-vivo validation of the implant performance}

We modeled the electric field in the retina generated by a subretinal $30 \mu \mathrm{m}$-thick array $1.5 \mathrm{~mm}$ in diameter with $40-\mu \mathrm{m}$ photovoltaic pixels, described in detail in ${ }^{10}$. In this array, each pixel has a SIROF-coated active electrode $18 \mu \mathrm{m}$ in diameter exposed to electrolyte. The return electrodes are connected to a SIROF-coated common return deposited at the periphery of the implant, as shown in Figure 3A. The electric field at any given time is a linear combination of a set of elementary fields, each of which corresponds to activation of one pixel individually, with coefficients equal to the currents at the respective active electrodes ${ }^{20}$. We generated the elementary electric fields of all pixels by numerically solving the Poisson equation of volume conduction, using the finite element method (FEM) in COMSOL Multiphysics 5.6 (COMSOL, Inc., Sweden), the model file of which is accessible online ${ }^{23}$. The boundary condition on the capacitive electrode-electrolyte interface of a SIROF electrode change from equipotential (EP) to a uniform current density $(U C D)^{24}$, with a time constant of $t \approx R C$, which is 0.24 $\mathrm{ms}$ for the active electrodes, and $40 \mathrm{~ms}$ for the return electrode. Since the pulse width in our measurements ranges from $4 \mathrm{~ms}$ to $10 \mathrm{~ms}$, we assumed the UCD boundary condition for the active electrodes and EP - for the return.

To model the spatial coupling of the electric potential between pixels, we generalized the access resistance of one electrode to the cross-resistance matrix $\mathbf{R}$ of all active electrodes, where the entry in row $i$ and column $j$ corresponds to the potential elevation at an active electrode $i$, caused by a unitary current injection from an active electrode $j^{20}$. The diagonal entries of $\mathbf{R}$ represent the access resistance of each active electrode. To compute the interrelated circuit dynamics of all pixels, we then generalized the equation of the nonlinear circuit dynamics of a photovoltaic pixel ${ }^{25}$ to the multi-dimensional form, as shown in Supplementary Materials. The multi-dimensional nonlinear differential equation was solved with our customized adaptive-step gradient descent method in MATLAB R2017a (MathWorks, MA), the source code of which is accessible online ${ }^{26}$. To compute the electric field in the retina, the elementary electric fields were linearly combined using coefficients obtained from the multi-dimensional circuit dynamics.

For validation of the spatiotemporal model, we placed the device in a Petri dish filled with Dulbecco's phosphate-buffered saline (DPBS). The 10-ms pulses of 880-nm laser light were projected on the device surface at a repetition rate of $40 \mathrm{~Hz}$ at intensity of $8 \mathrm{~mW} / \mathrm{mm}^{2}$. Micropipettes with a $5 \mu \mathrm{m}$ tip diameter were used to measure the electric potential $20 \mu \mathrm{m}$ above the device. To study the current dynamic under changing images, a field stop in the laser projection system swiftly closed between two laser pulses, reducing the illuminated area from full-field to an octagon covering about a quarter of the pixels (Figure 4A). Electric potential before and after the closure was modeled and compared to the measurement.

\section{Modeling the corneal signals and the in-vivo validation}

To model the electric potential generated by a subretinal implant on the cornea, we constructed an anatomically realistic model of the rat eye, as described by Selner et al. ${ }^{13}$, with adaptation for the thickness of a degenerate retina and a photovoltaic implant in the subretinal space. The corresponding COMSOL model file is accessible online ${ }^{23}$. We also incorporated a realistic geometry of the rat head to the model and computed the 
potential difference between the corneal and the nasal electrodes, as shown in Figure S4A in Supplementary Materials. Meshing and adjusting the complex geometry of the rat head was difficult and computationally slow, and since the initial result demonstrated that electric potential is confined to a few millimeters around the eye, we approximated the head geometry with a cylinder, while keeping all the ocular and orbital tissues the same as in the anatomically accurate model. This approximation resulted in a similar electric field, but with a more reliable meshing and adjustable geometry. Electric potential generated by a single activated pixel in such a simplified layout is shown In Figure 5A, overlaid with the diagram of the ocular anatomy. A more detailed diagram of the anatomical structures and the list of the tissue properties with the corresponding references are shown in the Supplementary Materials.

\section{Surgical procedures}

All experimental procedures were conducted in accordance with the Statement for the Use of Animals in Ophthalmic and Vision research of the Association for Research in Vision and Ophthalmology (ARVO) and approved by the Stanford Administrative Panel on Laboratory Animal Care. Royal College of Surgeons (RCS) rats were used as an animal model of the inherited retinal degeneration, and Long Evans (LE) rats were used as a control group with healthy natural vision. Animals are maintained at the Stanford Animal Facility under $12 \mathrm{~h}$ light/12h dark cycles with food and water ad libitum.

The planar photovoltaic devices were implanted in the subretinal space, as previously described ${ }^{27}$, typically at 6 months of age, after a complete loss of the outer nuclear layer, as evidenced by optical coherence tomography (OCT; HRA2-Spectralis; Heidelberg Engineering, Heidelberg, Germany). The implants were placed in the temporal-dorsal region, approximately $1 \mathrm{~mm}$ away from the optic nerve. A total of 9 animals were implanted with $1.5 \mathrm{~mm}$ diameter arrays containing pixels of $40 \mu \mathrm{m}(n=4)$ and $20 \mu \mathrm{m}(n=5)$. Animals were anesthetized with a mixture of ketamine $(75 \mathrm{mg} / \mathrm{kg}$ ) and xylazine $(5 \mathrm{mg} / \mathrm{kg})$ injected intraperitoneally.

To visualize the retina and the implant, animals were monitored over time using OCT. For measurements of the visually evoked potentials (VEP), each animal was implanted with three transcranial screw electrodes: one electrode over each hemisphere of the visual cortex ( $4 \mathrm{~mm}$ lateral from midline, $6 \mathrm{~mm}$ caudal to bregma), and a reference electrode ( $2 \mathrm{~mm}$ right of midline and $2 \mathrm{~mm}$ anterior to bregma).

\section{Measurements of the prosthetic visual acuity}

Following anesthesia and pupil dilation, the cornea was covered with a viscoelastic gel and a cover slip to cancel the cornea optical power and ensure good retinal visibility. The retinal implant was illuminated with a customized projection system, consisting of an $880 \mathrm{~nm}$ laser (MF-880 nm-400 $\mu \mathrm{m}$, DILAS, Tucson, AZ), customized optics, and a digital micromirror display (DMD; DLP Light Commander; LOGIC PD, Carlsbad, CA) for pattern projection. The entire optical system was integrated with a slit lamp (Zeiss SL-120; Carl Zeiss, Thornwood, NY) for real-time observation of the illuminated retina via a CCD camera (acA1300-60gmNIR; Basler, Ahrensburg, Germany).

Visual acuity was assessed by applying alternating gratings, where the NIR illumination was pulsed at 64 $\mathrm{Hz}$ and the grating patterns were switched at $1 \mathrm{~Hz}$. The grating bar width ranged from $13 \mathrm{~m}$ to $157 \mathrm{~m}$ on the retina while the light intensity was set to $1.2 \mathrm{~mW} / \mathrm{mm} 2$. The light intensity was measured before and after each measurement at the cornea and scaled by the ocular magnification squared, where magnification is defined as the ratio between the size of a projected square on the retina and in air. VEPs were recorded using the Espion E3 system (Diagnosys LLC, Lowell, MA) at a sampling rate of $2 \mathrm{kHz}$ and averaged over 250 trials. Corneal signal reflecting the device performance was simultaneously measured using ERG electrodes, relative to the reference electrode in the nose. The corneal signal was also used as a template to remove the device artifact in VEP waveforms. High frequency components in VEP traces were further filtered using a spectrum reconstruction method. Acuity was defined as an intersection of the logarithmic fitting line with the noise level. The VEP data that support the findings of this study are available from the corresponding author upon reasonable request.

\section{Measurements of the natural visual acuity}

For measurements of the natural acuity under visible light, we could not utilize the same system used for prosthetic vision because the latter was optimized for NIR light and exhibited strong chromatic aberrations at visible wavelengths, resulting in a significant loss of contrast in fine patterns. Therefore, we measured monocular natural acuity in LE rats $(n=5)$ with a conventional large white screen in angular units (cycle per degree), and then related it to the linear resolution $(\mu \mathrm{m})$ on the retina. 
Following anesthesia, the cornea was covered with a plano contact lens and animals were placed $23 \mathrm{~cm}$ away from a $40 \mathrm{~cm}$-wide white screen, providing luminance of $51 \mathrm{~cd} / \mathrm{m}^{2}$ on the cornea. Black-and-white rectangular-wave grating patterns of $50 \%$ duty cycle alternated at $1 \mathrm{~Hz}$, providing 500 ms exposure duration for each pattern. Stripe widths varied from 0.21 to $16.7 \mathrm{~mm}$ on the screen, corresponding to 9.6 to 0.12 cycles per degree (cpd) in the eye. VEP measurements were averaged over 100-150 cycles.

To relate the angular units of acuity (cpd) to the stripe width on the retina $(\mu \mathrm{m})$, we performed spot retinal photocoagulation (PASCAL laser, Topcon, San Jose, CA). Animals were anesthetized and mounted on a gonioscopic stage, and retinal laser burns were produced for 3 angular positions separated by 4 degrees each. The $577 \mathrm{~nm}$ wavelength laser with a spot diameter of $100 \mu \mathrm{m}$ was centered on the pupil for each exposure of $70 \mathrm{~ms}$ in duration at $70 \mathrm{~mW}$ of power (or two sequential pulses of $100 \mathrm{~ms}$ at $50 \mathrm{~mW}$ ). Retinal burns were then imaged together with the NIR grating pattern projected into the eye in our slit-lamp based VEP system used for the measurements of prosthetic acuity, as described above. Distance between the centers of the burns was measured using the NIR patterns as a scale bar. The resulting conversion factor was found to be $64.3 \pm 2.9 \mu \mathrm{m}$ per degree of the visual angle, very similar to the $65.3 \pm 6.7 \mu \mathrm{m} / \mathrm{deg}$ based on optical modeling of the rat eye ${ }^{28}$. The uncertainty range in this conversion is represented by horizontal error bars in Figure 7D. Logarithmic line was fit to the falling trend of the plot, excluding the edge data point corresponding to the maximum value. Acuity was defined as a crossing point of the fitting line with the noise floor.

\section{Acknowledgements}

Studies were supported by the National Institutes of Health (Grants R01-EY-027786 and P30-EY-026877), the Department of Defense (Grant W81XWH-19-1-0738), AFOSR (Grant FA9550-19-1-0402), Wu Tsai Institute of Neurosciences at Stanford, and unrestricted grant from Research to Prevent Blindness. Photovoltaic arrays were fabricated at the Stanford Nano Shared Facilities (SNSF) and Stanford Nanofabrication Facility (SNF), which are supported by the National Science Foundation award ECCS1542152. K.M. was supported by a Royal Academy of Engineering Chair in Emerging Technology, UK. We would like to thank Dr. Tong Ling for his help with the design and assembly of the optical system for prosthetic acuity measurements and its software.

\section{Author contributions statement}

B.-Y. Wang conducted the electrophysiological measurements, Z.C. Chen performed the computational modeling and ex-vivo characterization of the implant, M. Bhuckory performed the implantations and in-vivo imaging and helped with prosthetic acuity measurements, T. Huang, A. Shin and L. Galambos worked on fabrication of the implants under the guidance of T. Kamins and K. Mathieson. V. Zuckerman conducted natural acuity measurements and helped with prosthetic acuity measurements, E. Ho built the system and helped with the software development for natural acuity measurements. Ethan Rosenfeld worked on the computational model of the eye and head in COMSOL. D. Palanker guided the research and data analysis. All authors participated in writing the manuscript.

\section{Additional information}

D. P. and T. K. serve as consultants to Pixium Vision. D. P.'s patents related to retinal prostheses are owned by Stanford University and licensed to Pixium Vision. All other authors declare no financial interests.

\section{References}

1. Wong, W.L., et al. Global prevalence of age-related macular degeneration and disease burden projection for 2020 and 2040: a systematic review and meta-analysis. Lancet Glob Health 2, E106-E116 (2014).

2. Liew, G., Michaelides, M. \& Bunce, C. A comparison of the causes of blindness certifications in England and Wales in working age adults (16-64 years), 1999-2000 with 2009-2010. Bmj Open 4(2014). 
3. Kim, S.Y., et al. Morphometric analysis of the macula in eyes with geographic atrophy due to age-related macular degeneration. Retina 22, 464-470 (2002).

4. Humayun, M.S., et al. Morphometric analysis of the extramacular retina from postmortem eyes with retinitis pigmentosa. Invest Ophth Vis Sci 40, 143-148 (1999).

5. Palanker, D., Le Mer, Y., Mohand-Said, S., Muqit, M. \& Sahel, J.A. Photovoltaic Restoration of Central Vision in Atrophic Age-Related Macular Degeneration. Ophthalmology 127, 1097-1104 (2020).

6. Edwards, T.L., et al. Assessment of the Electronic Retinal Implant Alpha AMS in Restoring Vision to Blind Patients with End-Stage Retinitis Pigmentosa. Ophthalmology 125, 432-443 (2018).

7. Palanker, D., Le Mer, Y., Mohand-Said, S. \& Sahel, J.-A. Simultaneous Perception of Prosthetic and Natural Vision in AMD Patients. (Research Square, https://www.researchsquare.com, 2021).

8. Loudin, J.D., et al. Optoelectronic retinal prosthesis: system design and performance. Journal of Neural Engineering 4, S72-S84 (2007).

9. Flores, T., et al. Honeycomb-shaped electro-neural interface enables cellular-scale pixels in subretinal prosthesis. Sci Rep 9, 10657 (2019).

10. Huang, T.W., et al. Vertical-junction photodiodes for smaller pixels in retinal prostheses. J Neural Eng (2021).

11. Chen, Z.C., Wang, B.Y. \& Palanker, D. Real-Time Optimization of the Current Steering for Visual Prosthesis. in 2021 10th International IEEE/EMBS Conference on Neural Engineering (NER) 592-596 (2021).

12. Werginz, P., Wang, B.-Y., Chen, Z.C. \& Palanker, D. On optimal coupling of the 'electronic photoreceptors' into the degenerate retina. Journal of Neural Engineering 17, 045008 (2020).

13. Selner, A.N., Derafshi, Z., Kunzer, B.E. \& Hetling, J.R. Three-Dimensional Model of Electroretinogram Field Potentials in the Rat Eye. IEEE Trans Biomed Eng 65, 2781-2789 (2018).

14. Harnois, C., Bodis-Wollner, I. \& Onofrj, M. The effect of contrast and spatial frequency on the visual evoked potential of the hooded rat. Exp Brain Res 57, 1-8 (1984).

15. Hamilton, R., et al. VEP estimation of visual acuity: a systematic review. Documenta ophthalmologica. Advances in ophthalmology 142, 25-74 (2021).

16. Boyes, W.K. \& Dyer, R.S. Pattern reversal visual evoked potentials in awake rats. Brain Res Bull 10, 817-823 (1983).

17. Kharauzov, A.K., et al. Objective measurement of human visual acuity by visual evoked potentials. Neurosci Behav Physiol 36, 1021-1030 (2006).

18. Lorach, H., et al. Photovoltaic restoration of sight with high visual acuity. Nature Medicine 21, 476-482 (2015).

19. Ho, E., et al. Characteristics of prosthetic vision in rats with subretinal flat and pillar electrode arrays. J Neural Eng 16, 066027 (2019).

20. Chen, Z.C., Wang, B. \& Palanker, D. Real-Time Optimization of the Current Steering for Visual Prosthesis. in IEEE EMBS NER'21 (2021).

21. Alexander, R.G., Macknik, S.L. \& Martinez-Conde, S. Microsaccade Characteristics in Neurological and Ophthalmic Disease. Front Neurol 9, 144 (2018).

22. Ko, H.K., Snodderly, D.M. \& Poletti, M. Eye movements between saccades: Measuring ocular drift and tremor. Vision Res 122, 93-104 (2016).

23. Chen, Z.C. COMSOL models for the paper "Electronic "photoreceptors" enable prosthetic vision with acuity matching the natural resolution in rats". 5081278 (Zenodo.org, 2021).

24. Chen, Z., Ryzhik, L. \& Palanker, D. Current Distribution on Capacitive Electrode-Electrolyte Interfaces. Physical Review Applied 13, 014004 (2020). 
25. Boinagrov, D., et al. Photovoltaic Pixels for Neural Stimulation: Circuit Models and Performance.

26. Chen, Z.C. CCCharlesChan/RP_multidim_circuit_solver: Retinal Prosthesis Multi-Dim Circuit Solver v1.0. 5081286 (Zenodo.org, 2021).

27. Lorach, H., et al. Performance of photovoltaic arrays in-vivo and characteristics of prosthetic vision in animals with retinal degeneration. Vision Res 111, 142-148 (2015).

405

406

407

28. Lozano, D.C. \& Twa, M.D. Development of a rat schematic eye from in vivo biometry and the correction of lateral magnification in SD-OCT imaging. Invest Ophthalmol Vis Sci 54, 6446-6455

408 (2013).

409

Figures

A

B

\section{NFL}

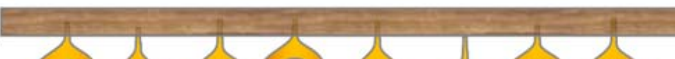

GC

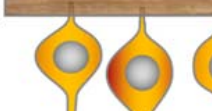

AC

AC

$\mathrm{HC}$

0

1
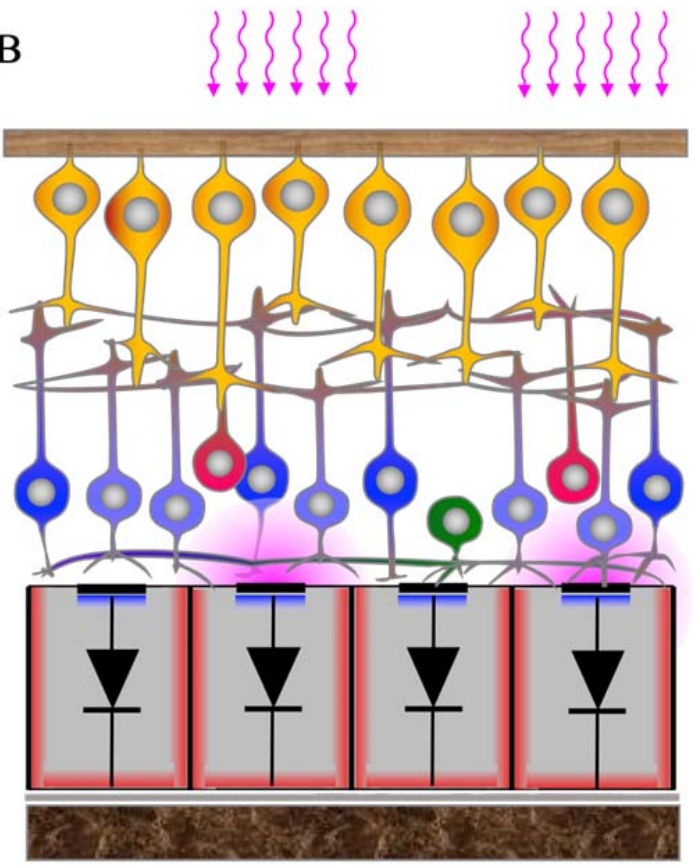

Figure 1. A) Diagram of the cellular layers in healthy retina, including the Choroid (Ch), Retinal Pigmented Epithelium (RPE), Photoreceptors (PR), Horizontal cells (HC), Bipolar cells (BC), Amacrine cells (AC), Inner Plexiform Layer (IPL), and Ganglion cells (GC), whose axons comprise the Nerve Fiber layer (NFL). B) Degenerate retina with a subretinal prosthesis composed of $20 \mu \mathrm{m}$ wide and $30 \mu \mathrm{m}$ deep photovoltaic pixels, which convert incoming light into electric current flowing through the tissue and polarizing the nearby neurons. 


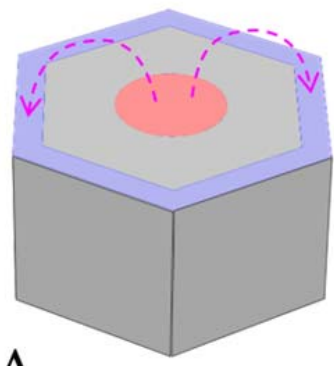

A

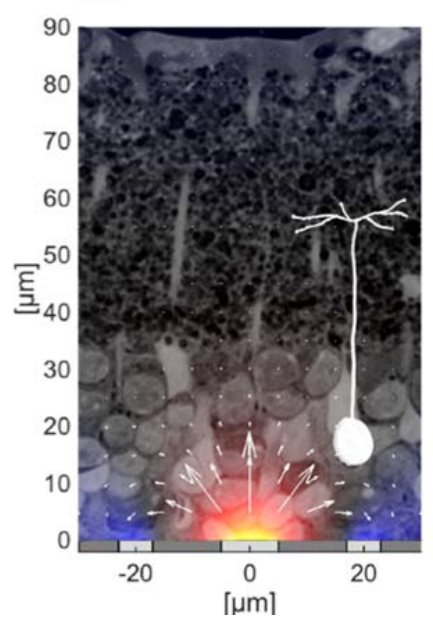

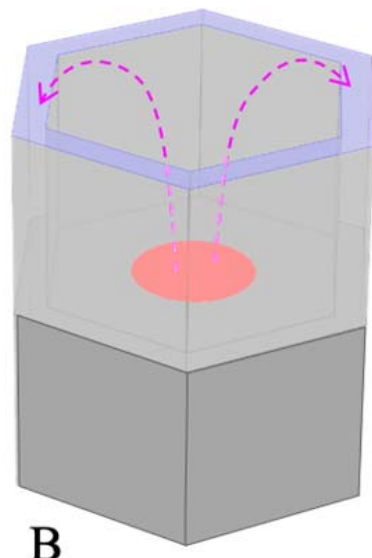

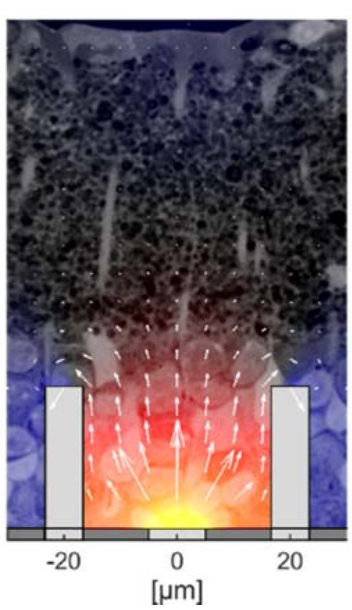

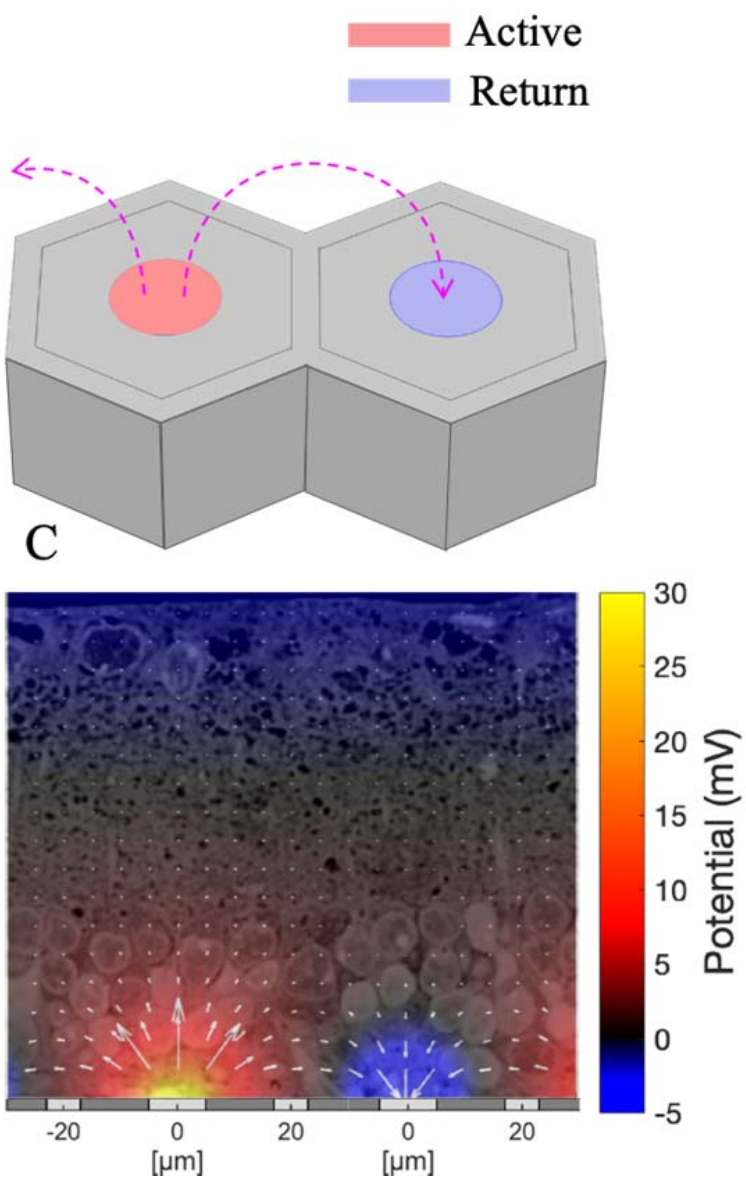

Active

Figure 2. Electric field above the photovoltaic pixels ( $40 \mu \mathrm{m}$ in width) of various geometries. A) A bipolar pixel containing a central active and a circumferential return electrode. (B) A honeycomb-shaped pixel with elevated return electrode. (C) Two monopolar pixels without local returns, where the active electrode of the left pixel acts as an anode and the one on the right - as a cathode, collecting $75 \%$ of the anodal current. Remaining current is collected by a global return around the edge of the device. Bottom panels show the corresponding simulated electric fields overlaid on a histology image of a degenerate rat retina (current $=68 \mathrm{nA}$ ). Diagram of a bipolar cell with its axonal terminals in the middle of IPL in the left panel illustrates its position and size with respect to the field penetration depth. The arrows represent current magnitude on a log scale. 

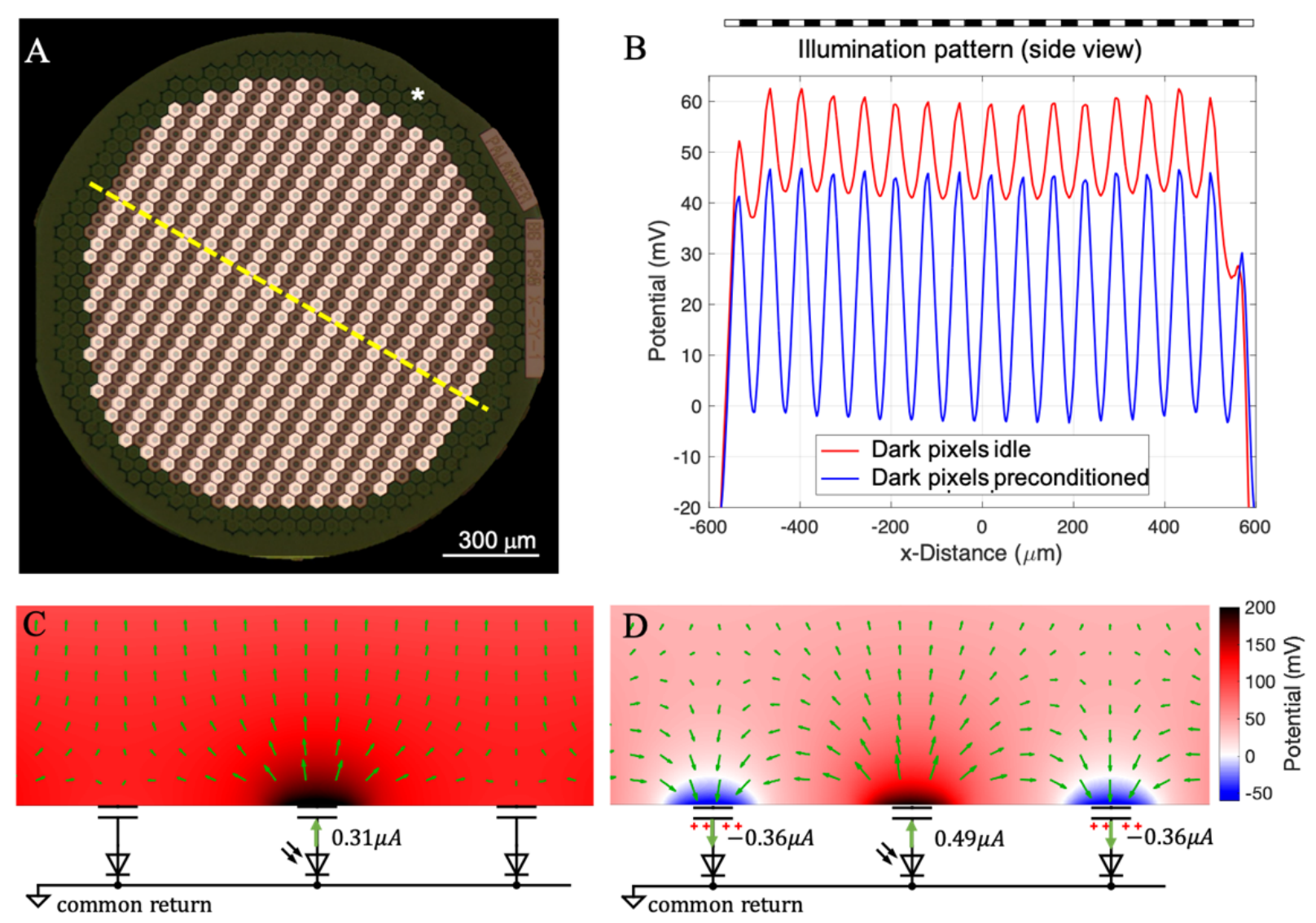

Figure 3. Electric field in electrolyte generated by the grating pattern with and without preconditioning of the dark pixels. A) Photovoltaic array of $1.5 \mathrm{~mm}$ in diameter composed of monopolar pixels of $40 \mu \mathrm{m}$ width, with a common return electrode (indicated by *) along the edge of the implant. B) Electric potential in electrolyte $10 \mu \mathrm{m}$ above the implant relative to the middle of IPL $(57 \mu \mathrm{m})$, plotted along the dash line shown in (A), when the dark pixels are non-conductive (red) and preconditioned (blue). C, D) Magnified view of the electric potential and current in the medium for the two plots in B. Length of the arrows represents the 0.6 power of the magnitude of the current density for visualization. 

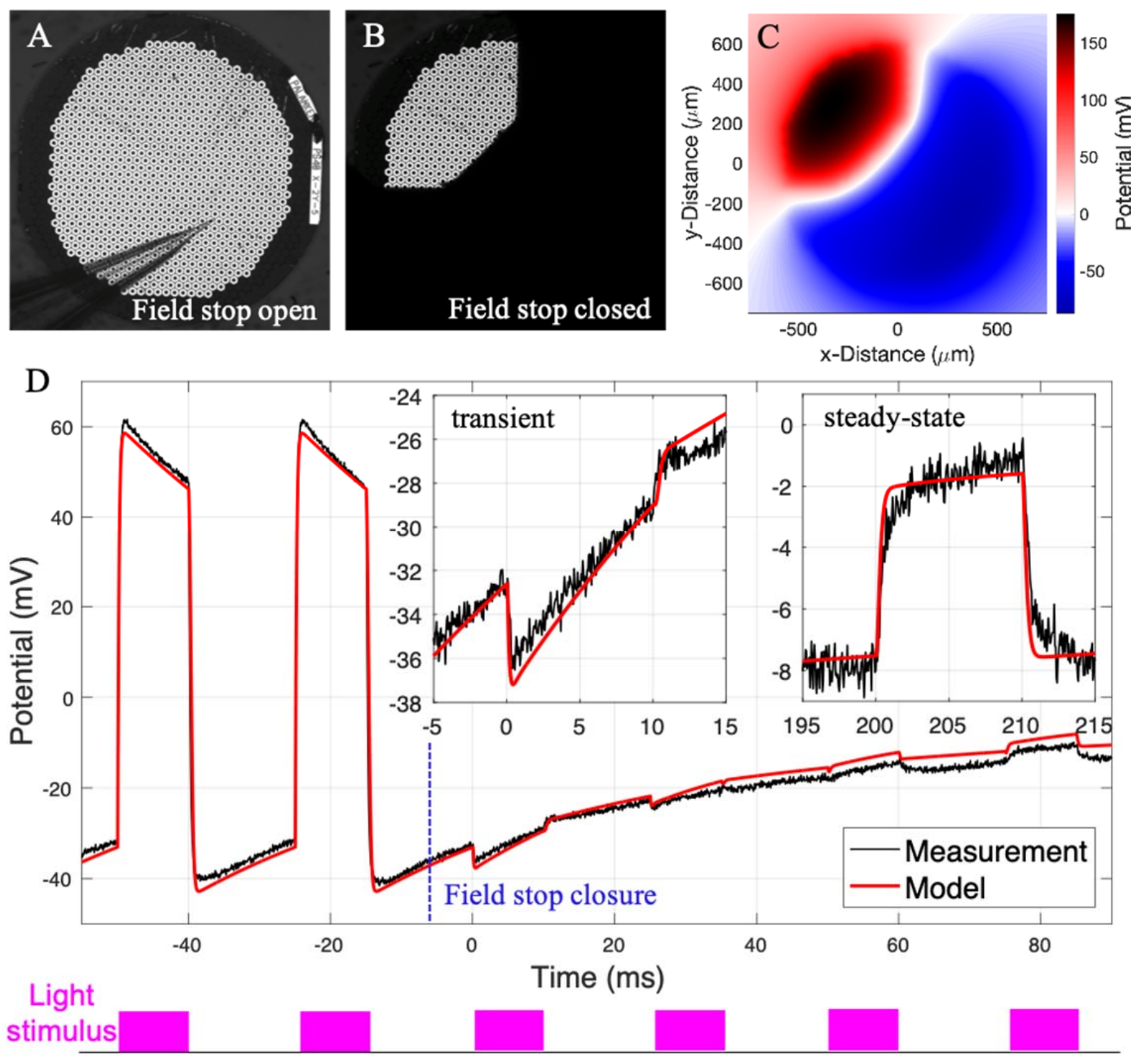

\section{stimulus}

Figure 4. Near-field electric potential in electrolyte $20 \mu \mathrm{m}$ above the photovoltaic array. A) View of the array and a pipette electrode under full-field illumination. B) The partially illuminated array after closure of the field stop. The pipette is at the same location, in the shaded zone. C) Map of the electric potential at $0.6 \mathrm{~ms}$. D) Electric potential induced by $10 \mathrm{~ms}$ pulses of NIR light before $(<0 \mathrm{~ms})$ and after $(>0 \mathrm{~ms})$ the decrease of the illuminated area. 

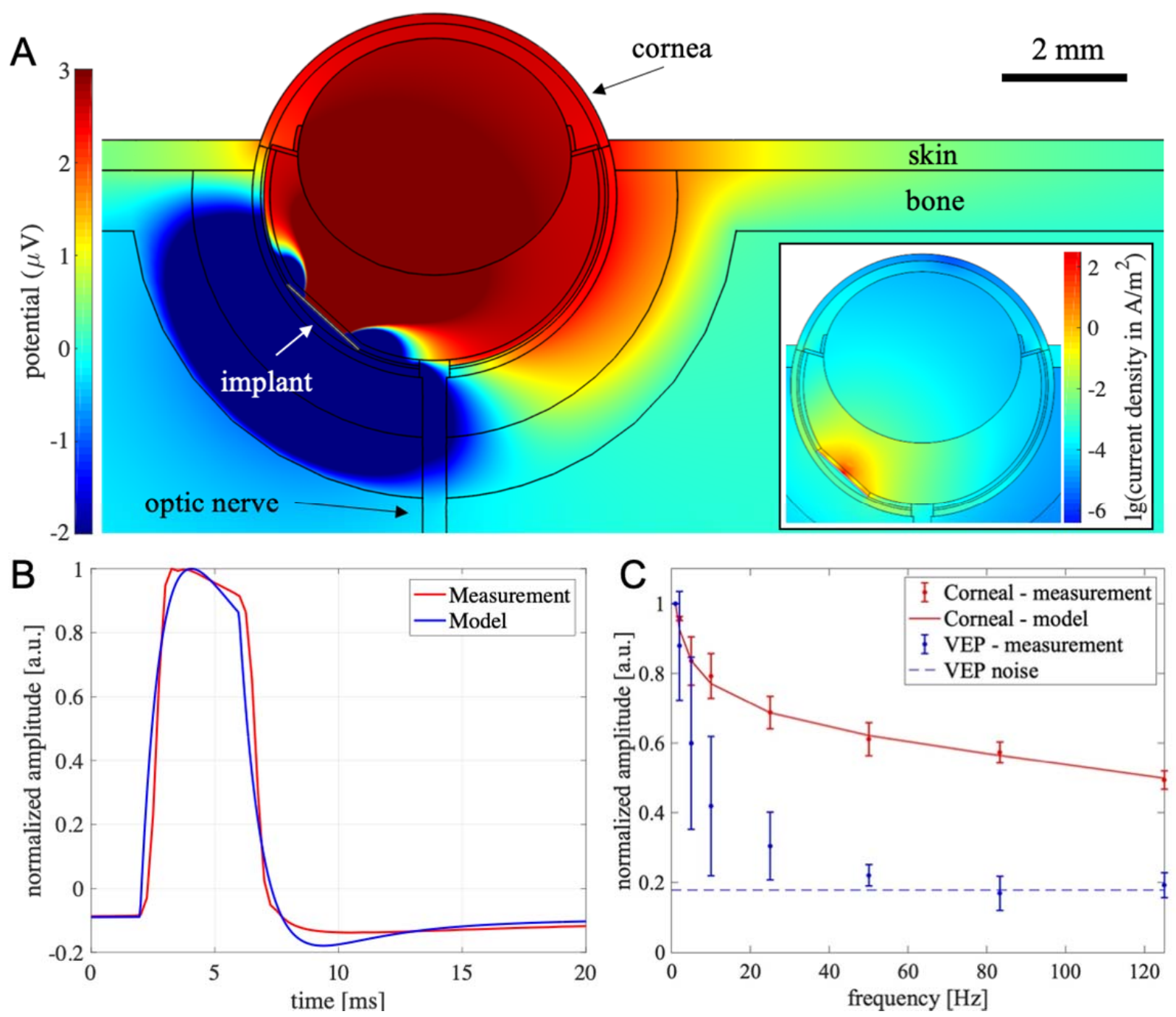

Figure 5. Far-field electric potential in the eye: measurements and modeling. (A) Finite element model (FEM) of a rat eye and orbital tissues, with a subretinal photovoltaic implant having $40 \mu \mathrm{m}$ pixels. Electric potential generated by one electrode injecting $1 \mu \mathrm{A}$ of current (an elementary field), shown in a color scale optimized for the corneal potential. An inset shows the current density on a logarithmic scale. (B) Measured and modeled corneal potential generated by a $4 \mathrm{~ms}$ pulse at $1.2 \mathrm{~mW} / \mathrm{mm}^{2}$ full-field illumination. (C) Peak-to-peak amplitude of the corneal signals induced by $4 \mathrm{~ms}$ pulses at repetition rates varying from 1 to $125 \mathrm{~Hz}$ under full-field illumination. Modeling result is shown by a line. 


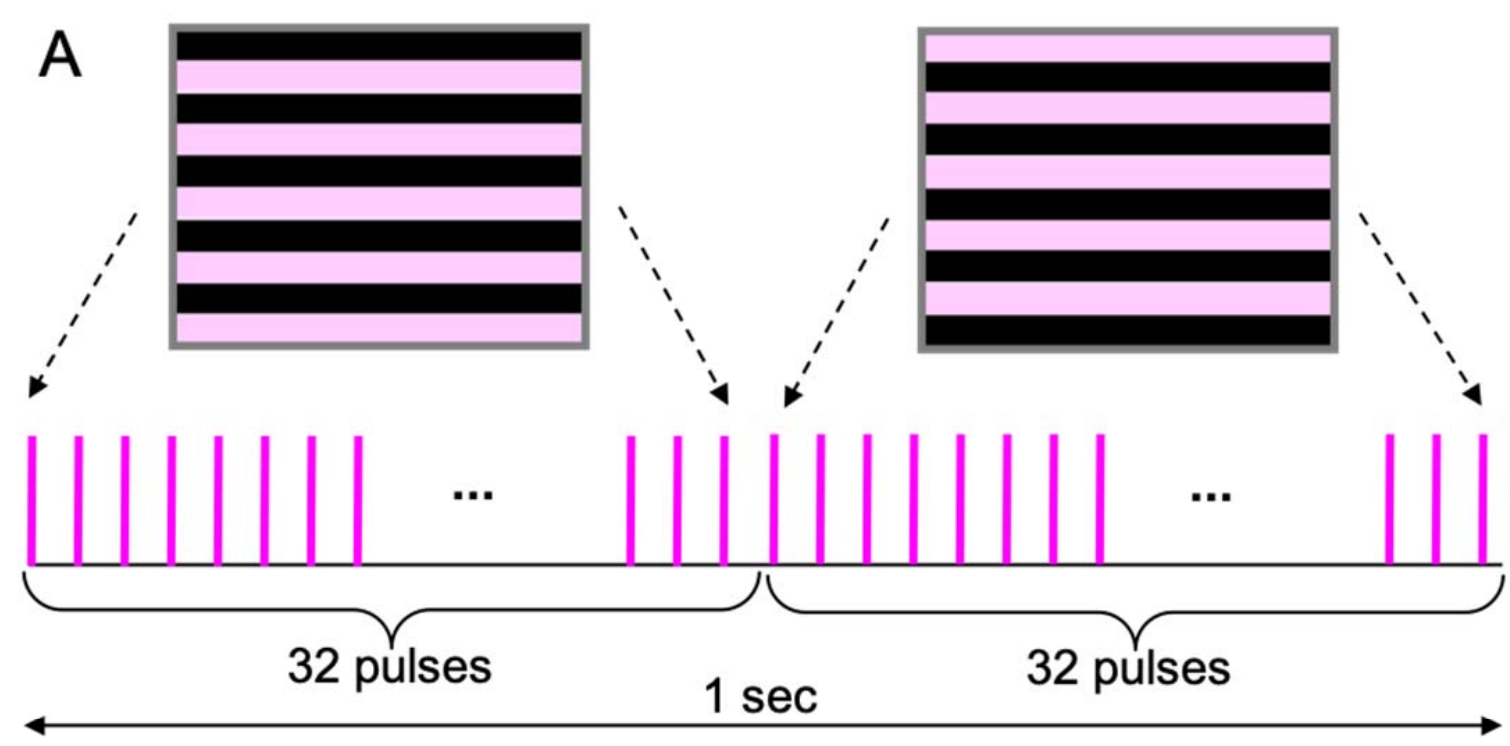

B
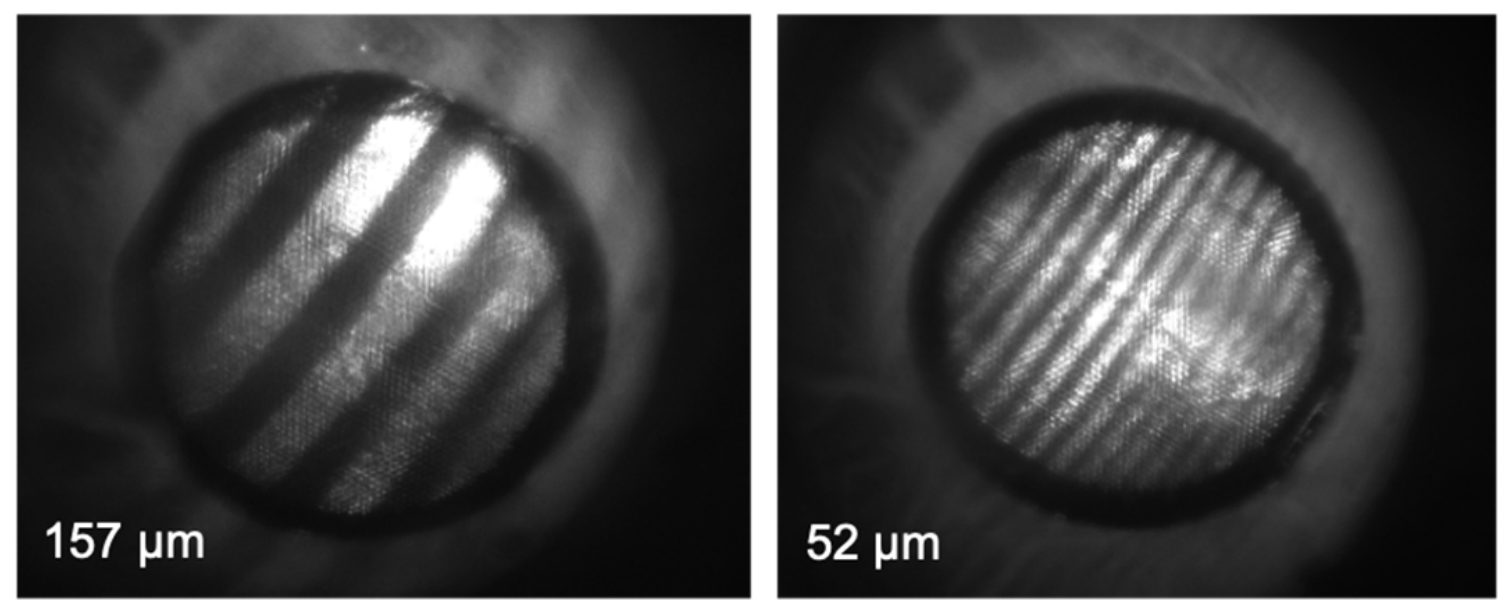

Figure 6. Measurements of the grating acuity. A: Projected grating patterns alternate at $1 \mathrm{~Hz}$, pulsed at a carrier frequency of $64 \mathrm{~Hz}$, with a pulse duration of $4 \mathrm{~ms}$. B: Images of the projected gratings with a bar widths of 157 and $52 \mu \mathrm{m}$, respectively, on a $20 \mu \mathrm{m}$ implant in a rat eye. 
A

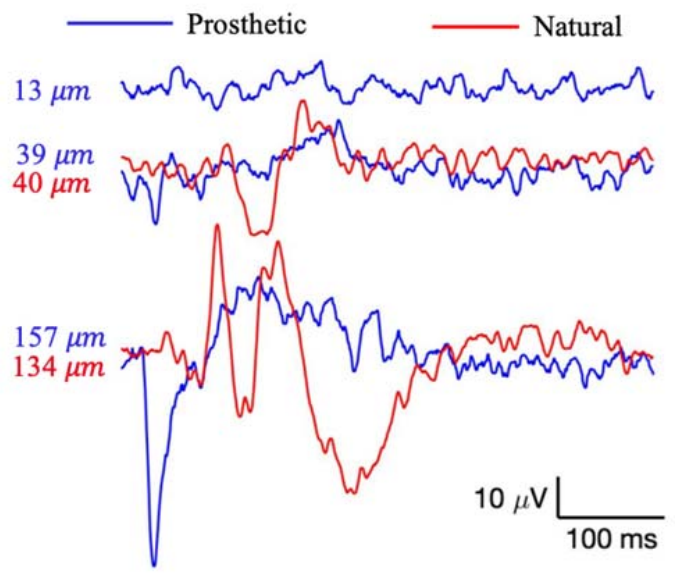

C

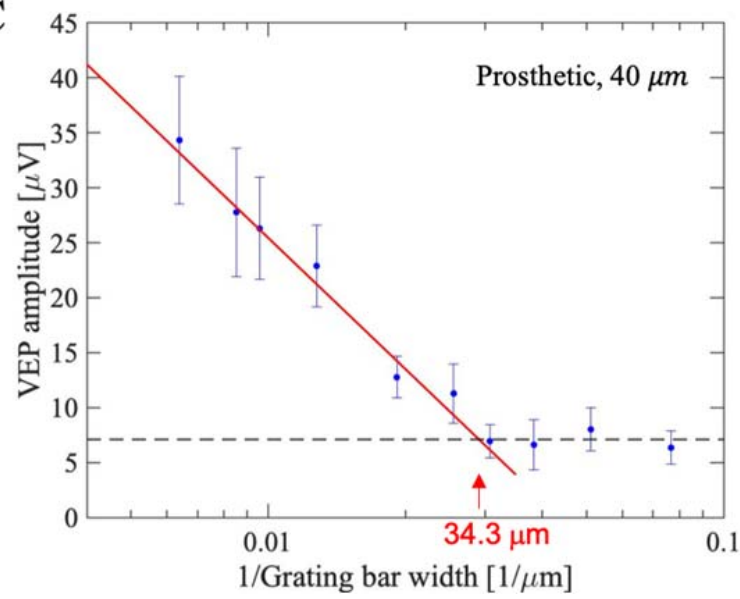

B

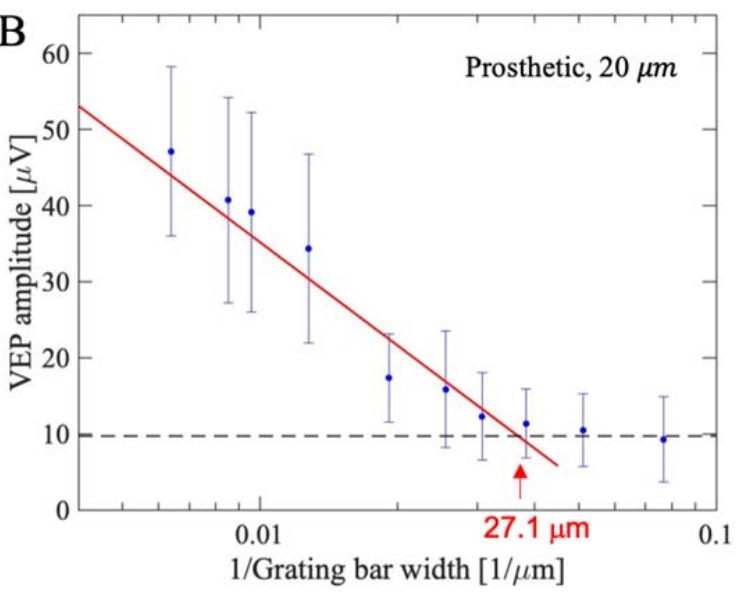

D

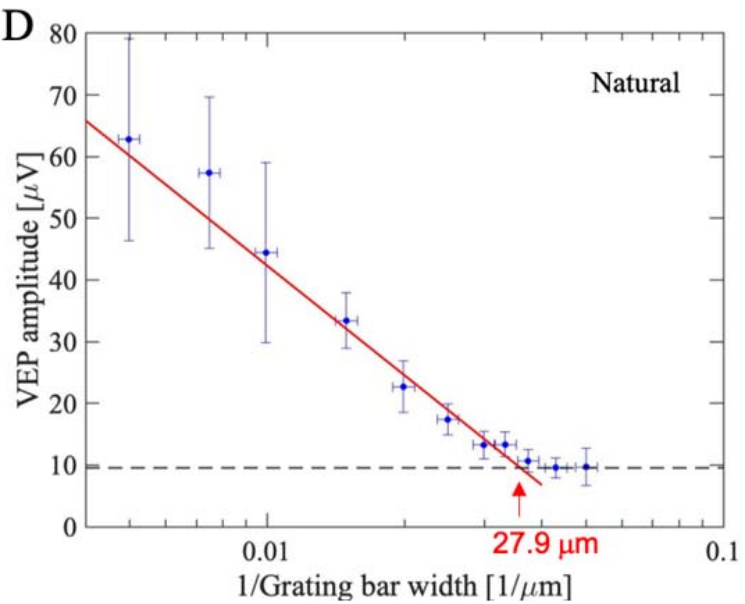

Figure 7. Prosthetic and natural visual acuity in rats. A) Example VEP waveforms in response to alternating gratings in RCS rats with implants and in LE rats. B-D) VEP amplitude as a function of the grating density (in units of the inverse bar width, $1 / \mu \mathrm{m}$ ) for prosthetic vision with $20 \mu \mathrm{m}$ pixels (B), $40 \mu \mathrm{m}$ pixels (C), and for natural vision (D). The vertical error bars indicate the standard deviations across animals, while the horizontal error bars in (D) represent the uncertainty in conversion from angular units of resolution (cpd) to linear bar width on the retina $(\mathrm{mm})$ (see Methods for further details). 